Article

\title{
Universes Inside a Black Hole with the de Sitter Interior
}

\section{Irina Dymnikova ${ }^{1,2}$}

1 A.F. Ioffe Physico-Technical Institute of the Russian Academy of Sciences, Polytekhnicheskaja 26, St. Petersburg 194021, Russia; igd.ammp@mail.ioffe.ru or irina@uwm.edu.pl

2 Department of Mathematics and Computer Science, University of Warmia and Mazury, Słoneczna 54, 10-710 Olsztyn, Poland

Received: 23 March 2019; Accepted: 7 May 2019; Published: 10 May 2019

Abstract: We outline the basic ideas and analyze the possibilities of the quantum birth of universes inside regular black holes with the de Sitter interior replacing a singularity. We compare different cases and show that the most plausible case is the birth of a flat universe from an initial quantum fluctuation with a small admixture of radiation and strings with the negative deficit angle, which provides the existence of a potential barrier needed for quantum tunneling.

Keywords: baby universes; regular black holes; de Sitter vacuum

\section{Introduction}

The idea of a de Sitter vacuum replacing a singularity has been suggested as early as 1966 by Sakharov, who considered $p=-\rho$ as one of the possible equations of state at super-high densities [1], and by Gliner who interpreted $p=-\rho$ as the equation of state of a vacuum with a non-zero density and assumed that it could be a final state in a gravitational collapse [2].

In the 1980s, several solutions were proposed on the basis of direct matching the de Sitter metric to the Schwarzschild metric on a spacelike junction surface $\Sigma_{0}$ of the Planckian thickness ([3] and references therein). The global structure of spacetime is shown in Figure 1, where $r_{g}=2 G M$ is the Schwarzschild horizon, and $r_{0}=\sqrt{3 / \Lambda}$ is the de Sitter horizon.

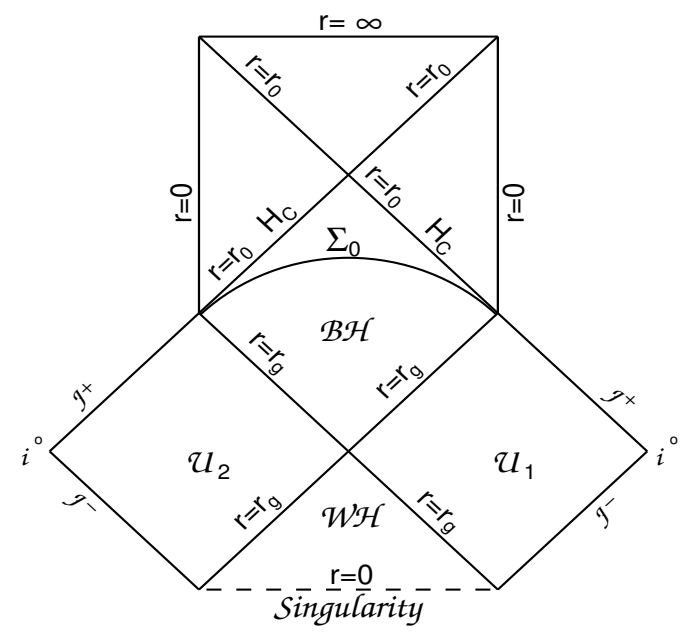

Figure 1. Penrose-Carter diagram for the case of the direct de Sitter-Schwarzschild matching. $\mathcal{J}^{-}$and $\mathcal{J}^{+}$are the past and future null (photon) infinities, while $i^{0}$ is the spacelike infinities. 
In 1988, Poisson and Israel assumed that transition to the de Sitter vacuum deeply inside the Schwarzschild black hole can result from the self-regulation of geometry by the vacuum polarization effects [3]. They analyzed such a transition in detail and found that in the resulting geometry there exists the Cauchy horizon $\left(H_{C}\right.$ in Figure 1$)$.

The idea of arising a baby universe inside a black hole has been investigated by Farhi and Guth in 1987 [4] as the idea of creation of a universe in the laboratory from a false vacuum bubble in the Minkowski space. An expanding bubble of the de Sitter vacuum generates the Schwarzschild geometry outside, separated by a thin wall from the de Sitter interior. The global structure of spacetime in this case implies that the arising of a baby universe must be associated with an initial spacelike singularity, which evidently represents a singular initial value configuration. Therefore, Farhi and Guth concluded that the initial singularity would be an unavoidable obstacle to the creation of a universe in the laboratory [4].

In 1990 Farhi, Guth, and Guven proposed the model with the little initial bubble arising without a singularity [5]. Quantum effects allow it to tunnel into the larger bubble which disappears beyond the black hole horizon of an outside observer, whereas on the inside region the bubble would classically evolve towards a new universe [5]. Independently, the question of a new universe inside a black hole has been addressed by Frolov, Markov, and Mukhanov in 1989 [6] in the framework of the hypothesis of the existence of the limiting curvature of the Planckian scale with the equation $p=-\rho$, and taking into account that the existence of the Cauchy horizon implies the absence of a global Cauchy surface. In 1990, they presented the analysis of a smooth de Sitter-Schwarzschild transition, including the case of the arising of a closed world in a new universe in the absolute future of the original universe, and the possibility of a white hole arising [7].

Later, the appearance of a new universe inside a black hole has been considered by Poplawski [8] in the Einstein-Cartan-Sciama-Kibble theory of gravity with torsion [9], where the spin induces gravitational repulsion at extremely high densities, which prevents the collapse to a singularity and provides formation of a de Sitter core and the appearance of a closed universe.

Oshita and Yokoyama [10] studied appearance of an inflationary universe beyond a wormhole throat, as initiated by a phase transition with symmetry restoration inside a thin-wall bubble around an evaporating black hole [11]. In this model, the thin-wall boundary of a false vacuum bubble induces quantum tunneling to a wormhole-like configuration, with exponentially expanding space beyond the wormhole throat filled with the false vacuum, interpreted as creation of a new inflationary universe in the final stage of the black hole evaporation.

The comprehensive analysis of a universe production involving wormholes has been done by Ansoldi, Merali, and Guendelman [12] on the basis of the causal structure of spacetime and connection between vacuum decay and inflation, and including the question of creation of a baby universe within our universe on the other side of a wormhole throat in future particle accelerators [13] or spontaneously.

The above models have also been based on the direct matching of the Schwarzschild and the de Sitter metrics using the thin shell approach (the global structure of spacetime is shown in Figure 1), which suggests that the whole dynamical evolution from the equation of state $p=\rho=0$ (the Schwarzschild sector) to $p=-\rho$ (the de Sitter sector) occurs within or nearby a very thin junction layer. The matched metrics typically have a jump at the junction surface [3].

The case of a distributed density profile $\rho(r)$ smoothly evolving from a large internal density of the de Sitter vacuum, arising due to symmetry restoration, has been studied in a general setting in [14], where it was found that solutions describing de Sitter-Schwarzschild transition belong to the class of solutions to the Einstein equations with the source term such that

$$
T_{r}^{r}=T_{t}^{t}\left(p_{r}=-\rho\right)
$$

The stress-energy tensor with the algebraic structure (1) describes a spherically symmetric vacuum invariant under boosts in the radial direction [14], which allows for an extension of the Einstein cosmological term $\Lambda g_{\mu v}$ to the spherically symmetric $r$-dependent cosmological term $\Lambda_{\mu v}$ [15]. 
In the case of de Sitter-Schwarzschild transition, it connects in a smooth way, the de Sitter vacuum $T_{\mu v}=(8 \pi G)^{-1} \Lambda g_{\mu v}$ replacing a singularity at the scale of symmetry restoration to the de Sitter group in the origin [16,17], and the Minkowski vacuum $T_{\mu \nu}=0$ at infinity.

Stress-energy tensors specified by (1) generate spacetimes with the de Sitter center [18], and represent a spatially inhomogeneous and time evolving vacuum dark energy related to spacetime symmetry (for a review, see [19]). In the spacetime with a nonzero cosmological constant, $\lambda$, they relate two de Sitter vacua and describe relaxation of a cosmological constant from the big value, needed for driving inflation, to a small finite value [20] (for a review [21]). Regular solutions to the Einstein equations belong to the Kerr-Schild class and describe regular cosmological models and compact objects generically related to dark energy via their de Sitter interiors-regular black holes, their remnants, and self-gravitating vacuum solitons which can be responsible for observational effects related to a dark matter (for a recent review, see [22]).

In Section 2, we outline the global structure of spacetime and the possibilities, additional to predicted in $[5,7]$, for the arising of universes inside black holes. Otherwise (as it is here) this phrase does not make the proper sense. The question of baby universes inside a regular black hole is addressed in Section 3. We present the analysis of the matter content of fluctuations which can provide the existence of a barrier needed for quantum birth of an open and flat universe. Possibilities for the quantum birth of an open, flat, and closed universe are compared, and it appears that the most plausible case is the birth of a flat $(\Omega=1)$ universe. In Section 4 we summarize the results.

\section{Regular Black Hole with the de Sitter Interior}

The metric generated by a source term specified by (1) belongs to the Kerr-Schild class [23] and can be written as [14]:

$$
d s^{2}=\left(1-\frac{R_{g}(r)}{r}\right) d t^{2}-\frac{d r^{2}}{1-\frac{R_{g}(r)}{r}}-r^{2} d \Omega^{2} ; R_{g}(r)=2 G \mathcal{M}(r),
$$

where $d \Omega^{2}$ is the line element on the unit two-sphere. The mass function $\mathcal{M}(r)$ reads:

$$
\mathcal{M}(r)=4 \pi \int_{0}^{r} \rho(x) x^{2} d x
$$

A density profile $\rho(r)$ should provide the proper asymptotic behavior of $R_{g}(r)$, including vanishing as $r \rightarrow \infty$ quick enough to guarantee finiteness of the total mass.

$$
M=4 \pi \int_{0}^{\infty} \rho(x) x^{2} d x<\infty ; \quad R_{g}(r \rightarrow \infty)=r_{g} ; \quad R_{g}(r \rightarrow 0)=\frac{r^{3}}{r_{0}^{2}} ; \quad r_{0}^{2}=\frac{3 c^{2}}{8 \pi G \rho_{0}},
$$

where $\rho_{0}$ is the vacuum density at $r=0$. In [14], the exact analytic solution has been found for the density profile $\rho(r)=\rho_{0} \exp \left(-r^{3} / r_{0}^{2} r_{g}\right)$, representing vacuum polarization in a spherical gravitational field estimated in the frame of a simple semiclassical model [17,24].

The spherically symmetric vacuum specified by (1) belongs to the Type I in the classification given by Hawking and Ellis [25]. For any source term $T_{\mu \nu}$ satisfying the weak energy condition $T_{\mu v} u^{\mu} u^{\nu} \geq 0$, for a timelike vector $u^{v}$, the density profile decreases monotonically ( $d \rho / d r \leq 0$ everywhere), since the weak energy condition holds for $\rho \geq 0, \rho+p_{k} \geq 0(k=1,2,3)$ [25].

The equation of state for the radial pressure follows from the algebraic structure of $T_{\mu \nu}(1)$, and the tangential pressure $p_{\perp}=-T_{\theta}^{\theta}=-T_{\phi}^{\phi}$ is calculated from the conservation equation $T_{j v}^{\mu v}=0$, yielding

$$
p_{r}=-\rho(r) ; p_{\perp}=-\rho(r)-\frac{r}{2} \frac{d \rho(r)}{d r} .
$$


Within the range of masses $M \geq M_{\text {crit }}$, where $M_{\text {crit }}$ corresponds to the double horizon, the de Sitter-Schwarzschild geometry (2) describes a regular black hole with the de Sitter interior [14,24], called a $\Lambda$ black hole $(\Lambda B H)$ in [26]. For $M>M_{\text {crit }}$ spacetime has two horizons, an event horizon $r=r_{+}$and an internal Cauchy horizon $r=r_{-}$. The global structure of spacetime is shown in Figure 2 (right) in comparison with the Schwarzschild spacetime (bottom-left).

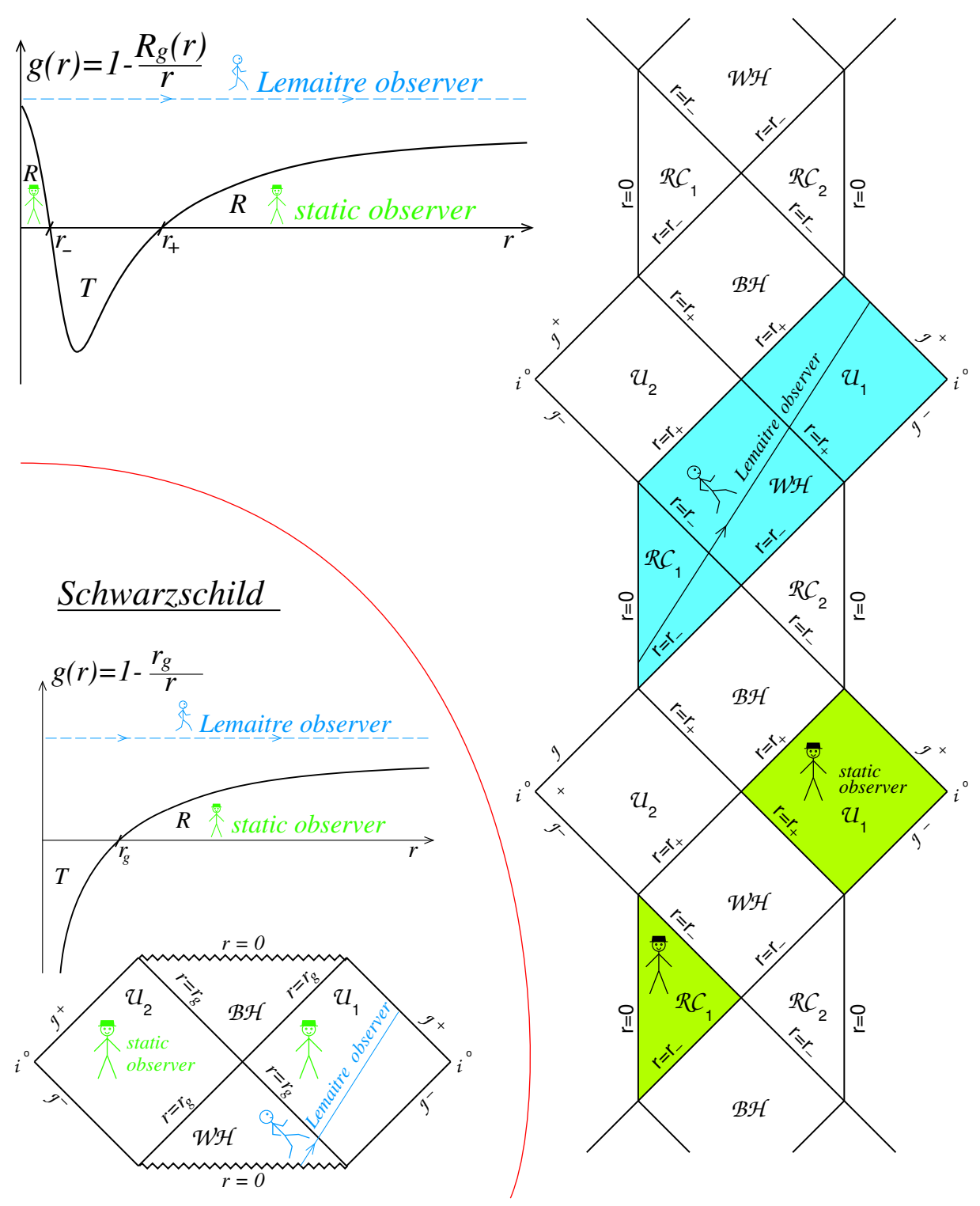

Figure 2. Penrose-Carter diagram of spacetime for a regular black hole.

It contains an infinite sequence of black and white holes $(\mathcal{B H}, \mathcal{W H})$ whose singularities are replaced with the future and past regular cores $\mathcal{R C}$ asymptotically de Sitter as $r \rightarrow 0$, and asymptotically flat universes $\mathcal{U}$ [24]. The Penrose-Carter diagrams in Figure 2 are plotted in coordinates related to the photon radial geodesics. The surfaces $\mathcal{J}^{-}$and $\mathcal{J}^{+}$represent their past and future infinities. The horizons $r=r_{+}$and $r=r_{-}$are formed by the outgoing and incoming radial photon geodesics (the null generators of horizons). Static observers can exist in the R-regions $\mathcal{R C}\left(0 \leq r<r_{-}\right)$ and $\mathcal{U}\left(r_{+}<r<\infty\right)$. For observers moving along radial timelike geodesics (the Lemaitre observers in Figure 2) the whole regions $0 \leq r<\infty$ are available. 
It is known that a white hole can be described as a cosmological model (see, e.g., [27]). In the case of a Schwarzschild white hole it includes the spacelike singularity $r=0$, entirely open for outside observers (Figure 2, left down); for this reason the existence of white holes (in a singular version) has been forbidden by the cosmic censorship [28].

Replacing a Schwarzschild singularity with the de Sitter center transforms the spacelike singular surface $r=0$ in the past of a (singular) white hole, into the timelike regular surface $r=0$ (Figure 2, right), and rehabilitates a white hole in its regular version [26].

A cosmological model related to a regular white hole (segment $\mathcal{R C}, \mathcal{W H}, \mathcal{U}$ in Figure 2, right) belongs to the cosmological models of the Lemaitre class with the de Sitter origin (for a review [21]). The regular white hole (more precisely its $\mathcal{R C}$ region) models the initial stages of a regular cosmology with the initial inflationary stage [26].

It is evident that a new universe inside a regular black hole can emerge in any of an infinite number of $\mathcal{B H}$ regions with the de Sitter vacuum in their interiors. Geodesic structure of the de Sitter-Schwarzschild spacetime shows [29] that the possibility of traveling to other universes through a black hole, reported for the Reissner-Nordström and the Kerr geometry ([30] and references therein), does exist in the case of a regular black hole with the de Sitter interior [29].

In the next Section, we estimate the probability of the arising of a closed world in the future of a black hole (as proposed in [7]) in the spacetime with the global structure shown in Figure 2 [26]. We also consider the additional option of a multiple quantum birth of open and flat causally disconnected universes from the initial quantum fluctuations arising in the $\mathcal{R C}$ regions.

\section{Birth of Universes Inside a Black Hole}

In the general case of a $\Lambda$ black hole with a distributed density profile and global structure of spacetime shown in Figure 2, the region near the timelike de Sitter surface $r=0$ is part of the regular core $\mathcal{R C}$, and can be considered as a small spherical bubble with the de Sitter vacuum trapped inside [17], which can be a seed for the quantum birth of a closed universe. The global structure of the de Sitter-Schwarzschild spacetime enables the arising of a closed world in any of an infinite number of $\mathcal{B H}$ structures in the future of a black hole in the original universe. This case is illustrated by Figure 3 , left [26].

Global structure of spacetime for the regular black hole provides additional possibilities related to instabilities of the de Sitter vacuum with respect to the quantum birth of a universe from nonperturbative quantum fluctuations of the metric [31-38].

The regular cosmological model for a universe born from a quantum fluctuation in de Sitter space and evolving from the de Sitter stage to the radiation-dominated stage has been proposed in 1975 in [39], where we also noticed the possibility of the multiple birth of causally disconnected universes from the de Sitter background. In 1982, a similar possibility has been studied in detail by Gott III as the creation of open FLRW universes in the quantum barrier penetration in de Sitter space, taking into account the Gibbons-Hawking radiation from the de Sitter horizon [40]. The case of the arising of open universes from the de Sitter vacuum is illustrated by Figure 3, right: The events $E$ and $E^{\prime}$ are creations of the causally disconnected universes. The curved lines are the world lines of co-moving observers. At the spacelike surface $\mathrm{AB}$, the phase transition occurs from the inflationary to the radiation dominated stage [40].

In the case of a regular black hole, the region ECB in Figure 3, right corresponds to the region $\mathcal{R \mathcal { C } _ { 1 }}$ in Figure 2, right; and the region BFD corresponds to a part of the region $\mathcal{R C}_{2}$. The regions $\mathcal{R} \mathcal{C}_{1}$ and $\mathcal{R C}_{2}$ in the de Sitter-Schwarzschild geometry are causally disconnected (they can be connected by spacelike curves only). Birth of an open (and flat) baby universe inside a regular black hole $\mathcal{R C}$ can occur in some of the infinite number of $\mathcal{R C}$ regions inside a $\Lambda$ black hole [26], and looks as in the picture shown in Figure 3, right. 

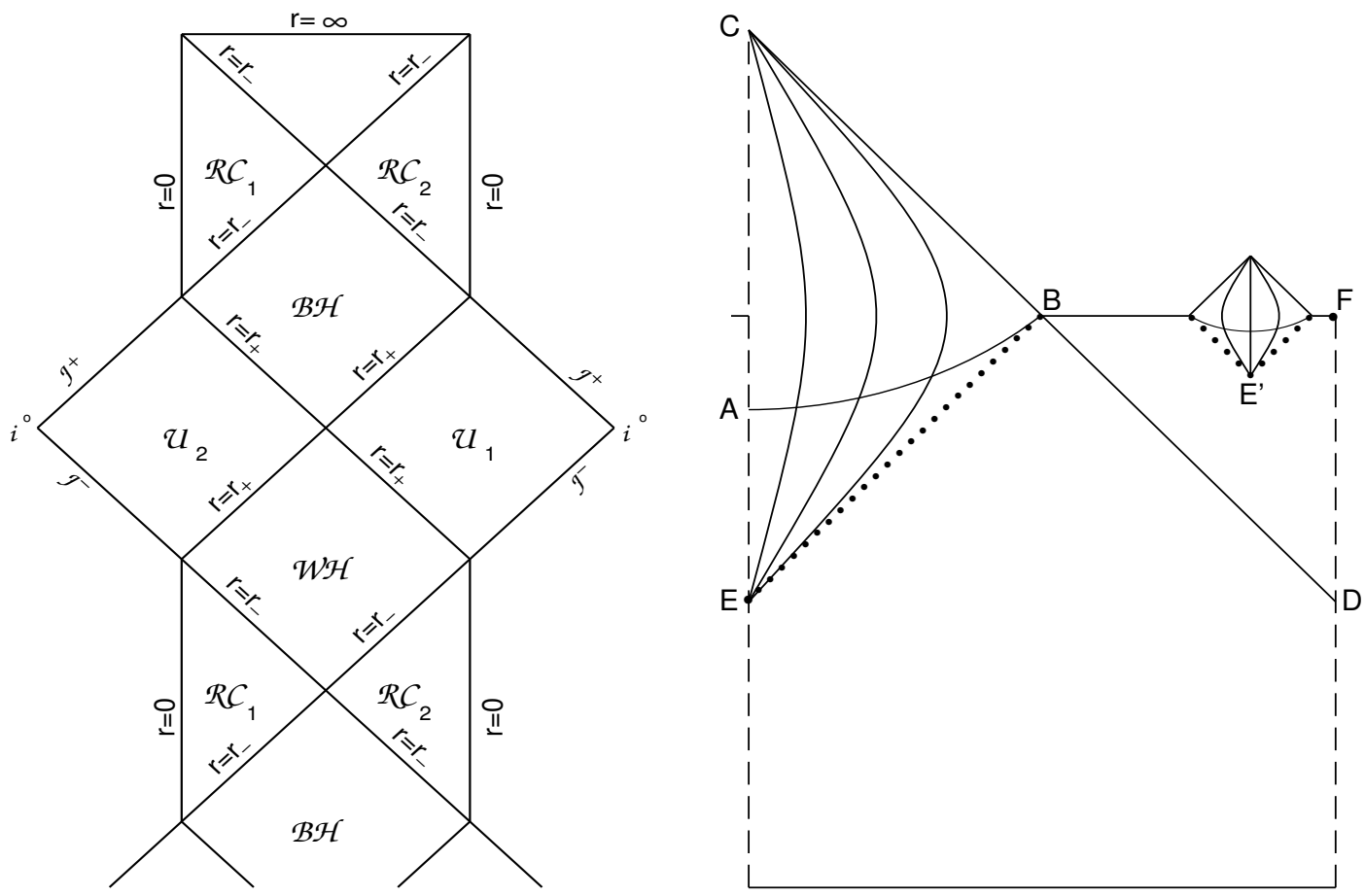

Figure 3. Left: Quantum birth of a closed universe inside a $\Lambda \mathrm{BH}$; Right: Penrose-Carter diagram [40] for the case of quantum birth of an open or flat universe.

In quantum cosmology, a nucleating bubble can be described by a minisuperspace model of the Wheeler-DeWitt formalism, in which the quantum state of a universe is described by a wave function $\Psi\left[g_{i k}(x), \phi_{m}(x)\right]$, defined on the superspace of all 3-dimensional geometries $g_{i k}(x)$ and the matter field configurations $\phi_{m}(x)$ [41-44]. The wave function of the universe satisfies the Wheeler-DeWitt second-order functional differential equation $\hat{H} \Psi=0$. For minisuperspace models, which have a finite number of degrees of freedom, derivation of the Wheeler-DeWitt equation from a path integral approach has been presented in $[45,46]$.

The Friedmann equation in the conformal time $a d \eta=c d t$, where $a$ is the scale factor, reads

$$
\left(\frac{d a}{d \eta}\right)^{2}=\frac{8 \pi G \rho a^{4}}{3 c^{2}}-k a^{2}
$$

where $k=0, \pm 1$ is the curvature parameter. The procedure of quantization $[32,35,45,46]$ gives the Wheeler-DeWitt equation in the minisuperspace for the wave function of an arising universe [32,45], which can be reduced to the Schrödinger equation [47]

$$
\frac{\hbar^{2}}{2 m_{P l}} \frac{d^{2} \psi}{d a^{2}}-U(a) \psi=0 ; U(a)=\frac{m_{P l} c^{2}}{2 l_{P l}^{2}}\left(k a^{2}-\frac{8 \pi G \rho a^{4}}{3 c^{2}}\right)
$$

The case of the arising of a de Sitter vacuum bubble in the Minkowski space, considered in [4,5], corresponds to the birth of a closed world from "nothing" [32,34], distinguished by the fact that its total energy is zero [27]. The minisuperspace model has two degrees of freedom-the scale factor and a homogeneous scalar field. A wave function satisfies the equation (7) and describes a tunneling from "nothing" to a de Sitter space, followed by an inflationary scenario. The potential in (7), appropriate for the arising of a closed world due to the existence of a barrier in the case of the positive curvature $k=1$, has the form

$$
U(a)=\frac{m_{P l} c^{2}}{2 l_{P l}^{2}}\left(a^{2}-\frac{a^{4}}{r_{0}^{2}}\right)
$$


and is shown in Figure 4, left. For the birth of a closed universe from "nothing" with the potential (8), the penetration factor is given by $[5,26,47]$

$$
D_{1}=\exp \left[-\frac{2}{3}\left(\frac{r_{0}}{l_{P l}}\right)^{2}\right]=\exp \left(-\frac{2}{3} \times 10^{16}\right)
$$

where the numerical estimate is given for the GUT scale $E_{G U T} \sim 10{ }^{15} \mathrm{GeV}$.

Inside a regular black hole with the interior de Sitter vacuum, its instability to nonperturbative quantum metric fluctuations can result in the quantum birth of a universe in some of the $\mathcal{R C}$ regions in the absolute future of an observer in the original universe. To calculate and compare probabilities for the birth of an open and flat universe, we have to extend the matter content to ingredients which can provide the existence of a barrier in the case of the negative $(k=-1)$ and zero $(k=0)$ curvature.

In the Equations (6) and (7), the density for each matter component evolves with $a$ as [47]

$$
\rho=\rho_{0} B_{w}\left(\frac{r_{0}}{a}\right)^{3(1+w)}
$$

where $w$ is a factor in the equation of state $p=w \rho$. To estimate the probability of a quantum birth of a new universe in a $\Lambda$ black hole, we consider an initial quantum fluctuation which contains some admixture of radiation with $p=\rho / 3$ and strings with $p=-\rho / 3$. In this case the total density in the initial fluctuation is [48]

$$
\rho=\rho_{0}+\rho_{0}\left(B_{s} \frac{r_{0}^{2}}{a^{2}}+B_{\gamma} \frac{r_{0}^{4}}{a^{4}}\right)
$$

where $B_{s}$ and $B_{\gamma}$ refer to the strings and radiation contributions in the Equation (7) [48].

Equation (7) can be written in the form [47]:

$$
\frac{\hbar^{2}}{2 m_{P l}} \frac{d^{2} \psi}{d a^{2}}-[U(a)-E] \psi=0,
$$

where the energy $E$, related to the contribution of radiation in the density (11), and the potential $U(a)$ in (7) for this case are given by

$$
U(a)=\frac{m_{p l} c^{2}}{2 l_{p l}^{2}}\left[\left(k-B_{s}\right) a^{2}-\frac{a^{4}}{r_{0}^{2}}\right] ; \quad E=\frac{B_{\gamma}}{2}\left(\frac{r_{0}}{l_{P l}}\right)^{2} E_{P l} .
$$

The key point is that a matter component with $p=-\rho / 3$ contributes to the curvature term, in particular, a matter with the negative contribution $B_{S}$ (for example, strings with a negative deficit angle) mimics the positive curvature behavior and provides the appearance in (7) of a barrier, in the cases $k=0$ and $k=-1$, which makes the quantum birth of an open or flat universe with a non-zero energy in a tunneling event possible [49]. This case is illustrated by Figure 4, right [47,48].
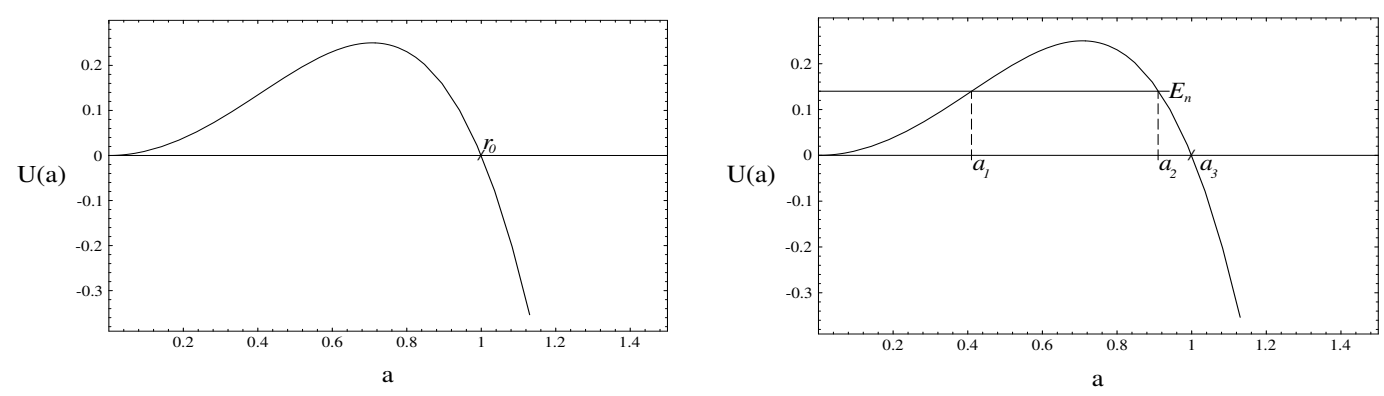

Figure 4. Left: The potential for the case of the birth of a closed universe from "nothing", Equation (8). Right: The potential for the case of the birth of a universe with a nonzero energy, Equation (13). 
Energy levels in the well are quantized $[47,48]$ in the WKB approximation $[47,50]$

$$
2 \int_{0}^{a_{1}} \sqrt{2 m_{P l}\left(E_{n}-U(a)\right)} d a=\pi \hbar(n+1 / 2) ; \quad E_{n} \simeq E_{P l} \sqrt{k-B_{s}}(n+1 / 2) .
$$

The probability of penetration through the potential barrier reads [50]

$$
D=\exp \left(-\frac{2}{\hbar}\left|\int_{a_{1}}^{a_{2}} \sqrt{2 m_{P l}[E-U(a)]} d a\right|\right) .
$$

This determines the probability of a tunneling, which describes the quantum growth of an initial bubble on its way to the classically permitted region. This formula, obtained in the frame of the Wheeler-DeWitt equation [26], coincides with the result obtained in the Euclidean approach [32,35,45] for the tunneling probability with the WKB wave function for the Euclidean Action written in the imaginary time in the Euclidean domain [35].

For the case of an initial fluctuation containing radiation and strings, Equation (13)

$$
D_{2}=\exp \left[-\frac{2}{3}\left(\frac{r_{0}}{l_{P l}}\right)^{2}\left(k-B_{s}\right)^{3 / 2}+(2 n+1)+I\right],
$$

where $I<10^{-2}(2 n+1)$, and analysis of the Bohr-Sommerfeld formula (14) near the maximum of the potential $U$, gives the general model constraint on $n$ [48]

$$
n+\frac{1}{2}<\frac{\left.\left(k-B_{S}\right)^{3 / 2}\right)}{\pi \sqrt{2}}\left(\frac{r_{0}}{l_{P l}}\right)^{2} .
$$

The observational constraint on the model parameter $\left(k-B_{s}\right)$ is needed to estimate the probability (16), for example by $\Delta T / T \leq 10^{-5}$, as in our Universe, restricts the parameter $\left(k-B_{s}\right)$ within the narrow range of around $10^{-6}$ [48]. Choosing some value from an admissible range, e.g., $\left(k-B_{s}\right) \simeq 3 \times 10^{-6}$, we find three possible cases for the matter content of the initial fluctuation in the quantum birth of a universe [48]:

(1) A closed universe, $k=1 ; B_{s} \simeq\left(1-3 \times 10^{-6}\right)$, born in the presence of strings or some other quintessence with $p=-\rho / 3$, whose density $\rho_{s}$ is comparable with the density of the de Sitter vacuum $\rho_{0}$ in (11).

(2) An open universe, $k=-1 ; B_{s} \simeq-\left(1+3 \times 10^{-6}\right)$, born due to the presence of strings with $p=-\rho / 3$ and the negative deficit angle, whose density $\rho_{s}$ is comparable with $\rho_{0}$.

(3) A flat universe, $k=0 ; B_{s} \simeq-3 \times 10^{-6}$, born from initial vacuum-dominated fluctuation due to the small admixture of strings with the negative deficit angle and the density $\rho_{s} \ll \rho_{0}$. This case is favored by the fact that the strings content is very small.

The probability of the birth of a universe with a non-zero energy $E_{n}$ from an initial fluctuation with the presence of strings is estimated as [48]

$$
D_{3}=\exp \left(-\frac{2}{3} \times 10^{7}\right)
$$

for all values of $k$. It appears substantially bigger (also in the case of the closed world with $k=1$ ) than the probability of a quantum birth of a closed universe from "nothing".

The most plausible case is the birth of a flat $(\Omega=1)$ universe distinguished by the fact that, in this case, the very small admixture of strings is sufficient.

Let us note that the existence of an infinite number of $\mathcal{R C}$ regions in the future of a $\Lambda \mathrm{BH}$ enhances the probability of arising baby universes inside it. 


\section{Conclusions}

The models with the direct matching of the Schwarzschild metric outside with the de Sitter metric inside correspond to the direct birth of a closed universe inside a black hole (Figure 1). In the general case of the de Sitter-Schwarzschild geometry with a smooth transition to interior de Sitter vacuum inside a black hole, the global structure of spacetime contains an infinite sequence of black and white holes with their future and past regular cores $\mathcal{R C}$ asymptotically de Sitter as $r \rightarrow 0$, and of asymptotically flat universes $\mathcal{U}$ in the future of a regular black hole (Figure 2, right). In this spacetime a quantum birth of a closed universe means closing this sequence in some of $\mathcal{B H}$ regions in the future of the original black hole (Figure 3, left).

In the case of a regular black hole with the de Sitter interior, the global structure of spacetime provides also the possibility of the multiple birth of flat and open causally disconnected universes in the $\mathcal{R C}$ regions (Figure 3, right), arising from initial fluctuations which contain strings with the negative deficit angle leading to the appearance of a potential barrier needed for quantum tunneling.

To evaluate the contribution of strings in calculating the probability of tunneling, we take into account decay of the inflationary vacuum into radiation within a nucleating bubble. Presence of radiation in the initial fluctuation corresponds to the quantum birth of a universe with a non-zero quantized energy, which is related to the strings content within an initial fluctuation. The observational constraint on the temperature anisotropy (known for our Universe) provides the restriction on the contribution of strings.

The probability of quantum birth of a universe is much bigger in this case than the probability of the birth of a closed universe from "nothing", for all three cases including an open, flat, and closed universe.

We compare different possibilities and show that the most plausible case is the birth of a flat universe $\left(\Omega=1\right.$ ) from an initial quantum fluctuation with a very small (of the order of $10^{-6}$ ) admixture of strings with the negative deficit angle.

The probability of the quantum birth of baby universes inside a $\Lambda$ black hole is not negligible, due to the existence of an infinite number of the appropriate $\mathcal{R C}$ regions inside a particular $\Lambda B H$. All this makes it possible to speculate (at least not exclude) that our Universe could be located inside some regular black hole with the de Sitter interior.

Funding: This research received no external funding.

Conflicts of Interest: The author declares no conflict of interest.

\section{References}

1. Sakharov, A.D. The Initial stage of an expanding universe and the appearance of a nonuniform distribution of matter. Sov. Phys. JETP 1966, 22, 241-249.

2. Gliner, E.B. Algebraic properties of the energy-momentum tensor and vacuum-like states of matter. Sov. Phys. JETP 1966, 22, 378-382.

3. Poisson, E.; Israel, W. Structure of the black hole nucleus. Class. Quant. Grav. 1988, 5, L201-L205. [CrossRef]

4. Fahri, E.; Guth, A. An obstacle to creating a universe in the laboratory. Phys. Lett. B 1987, 183, 149.

5. Farhi, E.; Guth, A.; Guven, J. Is it possible to create a universe in the laboratory by quantum tunneling? Nucl. Phys. B 1990, 339, 417-490. [CrossRef]

6. Frolov, V.P.; Markov, M.A.; Mukhanov, V.F. Through a black hole into a new universe? Phys. Lett. B 1989, 216, 272-276. [CrossRef]

7. Frolov, V.P.; Markov, M.A.; Mukhanov, V.F. Black holes as possible sources of closed and semiclosed worlds. Phys. Rev. D 1990, 41, 383-394. [CrossRef]

8. Poplawski, N.J. Universe in a black hole with spin and torsion. Astrophys. J. 2016, 832, 96-105.

9. Sciama, D.W. The Physical Structure of General Relativity. Rev. Mod. Phys. 1964, 36, 463-469. [CrossRef]

10. Oshita, N.; Yokoyama, J. Creation of an inflationary universe out of a black hole. Phys. Lett. B 2018, 785, 197-202. [CrossRef] 
11. Moss, I.G. Black-hole bubbles. Phys. Rev. D 1985, 32, 1333-1344. [CrossRef]

12. Ansoldi, S.; Merali, Z.; Guendelman, E.I. From black holes to baby universes: Exploring the possibility of creating a cosmos in the laboratory. Bulg. J. Phys. 2018, 45, 203-222.

13. Merali, Z. A Big Bang in a Little Room: The Quest to Create New Universes; Basic Books: New York, NY, USA, 2017.

14. Dymnikova, I. Vacuum nonsingular black hole. Gen. Rel. Grav. 1992, 24, 235-242. [CrossRef]

15. Dymnikova, I.G. The algebraic structure of a cosmological term in spherically symmetric solutions. Phys. Lett. B 2000, 472, 33-38. [CrossRef]

16. Dymnikova, I. Internal structure of nonsingular spherical black holes. In Internal Sructure of Black Holes and Spacetime Singularities; Burko, M., Ori, A., Eds.; Annals of the Israel Physical Society 13; Bristol In-t of Physics Pulishing: Bristol, UK; Philadelphia, PA, USA, 1997; pp. 422-440.

17. Dymnikova, I. The cosmological term as a source of mass. Class. Quant. Grav. 2002, 19, 725-739. [CrossRef]

18. Dymnikova, I. Spherically symmetric space-time with regular de Sitter center. Int. J. Mod. Phys. D 2003, 12, 1015-1034. [CrossRef]

19. Dymnikova, I. Dark energy and spacetime symmetry. Universe 2017, 3, 20. [CrossRef]

20. Dymnikova I,; Dobosz, A.; Sołtysek B. Lemaitre class dark energy model for relaxing cosmological constant. Universe 2017, 3, 39. [CrossRef]

21. Dymnikova I.; Dobosz, A. Spacetime symmetry and Lemaitre class dark energy models. Symmetry 2019, 11, 90. [CrossRef]

22. Dymnikova, I. Thermodynamics of horizons in regular spherical space-times of the Kerr-Schild class. Universe 2018, 4, 63. [CrossRef]

23. Kerr, R.P.; Schild, A. Some algebraically degenerate solutions of Einstein's gravitational field equations. Proc. Symp. Appl. Math. Am. Math. Soc. 1965, XVII, 199-207.

24. Dymnikova, I. De Sitter-Schwarzschild black hole: Its particlelike core and thermodynamical properties. Int. J. Mod. Phys. D 1996, 5, 529-540. [CrossRef]

25. Hawking, S.W.; Ellis, G.F.R. The Large Structure of Space-Time; Cambridge Univ. Press: Cambridge, UK, 1995.

26. Dymnikova, I.; Dobosz, A.; Filchenkov, M.; Gromov, A. Universes inside a $\Lambda$ black hole. Phys. Lett. B 2001, 506, 351-361. [CrossRef]

27. Landau, L.D.; Lifshitz, E.M. Classical Theory of Fields, 4th ed.; Butterworth-Heinemann: Oxford, UK, 1975.

28. Penrose R. General Relativity: An Einstein Centenary Survey; Hawking, S.W., Israel, W., Eds.; Cambridge Univ. Press: Cambridge, UK, 1979; p. 581.

29. Dymnikova, I.; Poszwa A.; Sołtysek B. Geodesic portrait of de Sitter-Schwarzschild spacetime. Grav. Cosmol. 2008, 14, 262-275. [CrossRef]

30. Novikov, I.D.; Frolov, V.P. Physics of Black Holes; Kluwer Academic Publishers: Dordrecht, The Netherlands, 1989.

31. Albrecht, A.; Steinhardt, P.J. Cosmology for Grand Unified Theories with radiatively induced symmetry breaking. Phys. Rev. Lett. 1982, 48. 1220-1223. [CrossRef]

32. Vilenkin, A. Quantum creation of universes. Phys. Rev. D 1984, 30, 509-511. [CrossRef]

33. Vilenkin, A. Quantum origin of the universe. Nucl. Phys. B 1985, 252, 141-152. [CrossRef]

34. Vilenkin, A. Boundary conditions in quantum cosmology. Phys. Rev. D 1986, 33, 3560-3569. [CrossRef]

35. Vilenkin, A. Approaches to quantum cosmology. Phys. Rev. D 1994, 50, 2581-2594. [CrossRef]

36. Dolgov, A.D.; Zel'dovich, Y.B.; Sazhin, M.V. Cosmology of the Early Universe; Nauka: Moscow, Russia, 1990.

37. Linde, A.D. Particle Physics and Inflationary Cosmology; CRC Press: London, UK, 1990.

38. Olive, K.A. Inflation. Phys. Rep. 1990, 190, 307-403. [CrossRef]

39. Gliner, E.B.; Dymnikova, I.G. Nonsingular Friedmann cosmology. Sov. Astr. Lett. 1975, 1, $93-95$.

40. Gott , J.R., III. Creation of open universes from de Sitter space. Nature 1982, 295, 304-307. [CrossRef]

41. DeWitt, B. Quantum Theory of Gravity. I. The Canonical Theory. Phys. Rev. 1967, 160, 1113-1148. [CrossRef]

42. DeWitt, B. Quantum Theory of Gravity. II. The Manifestly Covariant Theory. Phys. Rev. 1967, 162, 1195-1239. [CrossRef]

43. DeWitt, B. Quantum Theory of Gravity. III. Applications of the Covariant Theory. Phys. Rev. 1967, 162, 1239-1256. [CrossRef] 
44. Wheeler, J.A. Superspace and the Nature of Quantum Geometrodynamics. In Battelle Rencontres; Lectures in Mathematics and Physics; DeWitt, C.M., Wheeler, J.A., Eds.; Benjamin, W.A.: New York, NY, USA, 1967; pp. 242-308.

45. Hartle, J.B.; Hawking, S.W. Wave function of the Universe. Phys. Rev. D 1983, 28, 2960-2975. [CrossRef]

46. Halliwell, J.J. Derivation of the Wheeler-DeWitt equation from a path integral for minisuperspace models. Phys. Rev. D 1988, 38, 2468-2481. [CrossRef]

47. Dymnikova, I.; Filchenkov M. Quantum birth of a hot universe. Phys. Lett. B 2002, 545, 214-220. [CrossRef]

48. Dymnikova, I.; Filchenkov, M. Quantum origin of a hot universe. Int. J. Mod. Phys. D 2003, 12, 1197-1210. [CrossRef]

49. Filchenkov, M.L. The pre-de Sitter Universe in terms of quantum mechanics. Phys. Lett. B 1995, 354, $208-212$. [CrossRef]

50. Landau, L.D.; Lifshitz, E.M. Quantum Mechanics, 4th ed.; Butterworth-Heinemann: Oxford, UK, 1975.

(C) 2019 by the authors. Licensee MDPI, Basel, Switzerland. This article is an open access article distributed under the terms and conditions of the Creative Commons Attribution (CC BY) license (http://creativecommons.org/licenses/by/4.0/). 